\title{
Aproximaciones a la evaluación del desempeño en la gestión administrativa-financiera en las universidades públicas estatales
}

\author{
Salvador Francisco Tapia Spinoso a \\ Ana María Cuevas Padilla b
}

Resumen - La evaluación al desempeño en las universidades públicas estatales (UPE) es un tema prioritario en virtud de que éstas ejercen recursos de los presupuestos federal y estatal para el logro de sus fines académicos; es mandato constitucional que tales recursos públicos se administren con eficiencia, eficacia, economía, transparencia y honradez para satisfacer los objetivos a los que estén destinados. Por otro lado, la gestión administrativa-financiera en estas instituciones de educación se concreta a través de la estructura y funciones que dan soporte para el cumplimiento de sus propósitos, determinados en los ordenamientos jurídicos de cada entidad. Así, unidos el mandato constitucional y la misión de la institución, se delinean las directrices para la evaluación del desempeño.

Palabras clave - Desempeño Administrativo-Financiero, Indicadores, Universidades Públicas Estatales, Calidad.

Abstract - The performance evaluation of the state public universities (UPE) is a priority issue because they use resources from the federal and state budgets to achieve their academic goals; it is a constitutional mandate that such public resources are managed with efficiency, effectiveness, economy, transparency and honesty to satisfy the objectives for which they are earmarked. On the other hand, the administrative-financial management in these educational institutions is specified through the structure and the functions that provide support for the fulfillment of their purposes, which are determined in the legal systems of each entity. Thus, the constitutional mandate, together with the mission of the institution, outline the guidelines for the performance evaluation.

Keywords - Administrative-Financial Performance, Indicators, State Public Universities, Quality.

\section{CÓMO CITAR}

HOW TO CITE:

Tapia-Spinoso, S. F. y CuevasPadilla, A. M. (202I).

Aproximaciones a la evaluación del desempeño en la gestión administrativa-financiera en las universidades públicas estatales. Interconectando Saberes, (12), 63-7I.

https://doi.org/10.25009/is.v0il2 .2687

Recibido: 7 de diciembre de 2020

Aceptado: 6 de junio de 2021

Publicado: 20 de julio de 2021

${ }^{a}$ Universidad Veracruzana, México. E-mail: stapia@uv.mx

b Universidad Veracruzana, México. E-mail: anacuevas@uv.mx 


\section{INTRODUCCIÓN}

La Organización de las Naciones Unidas para la Educación, la Ciencia y la Cultura (UNESCO) ha manifestado que la educación es un derecho humano clave y fundamental que recoge los principios de indivisibilidad e interdependencia de todos los demás derechos humanos, ya que refiere condiciones civiles, políticas, económicas, sociales y culturales. Una implementación efectiva de este derecho es, efectivamente, un requisito ineludible para la participación de los ciudadanos en todos los aspectos de la vida, en un ámbito de justicia y democracia (Organización de las Naciones Unidas para la Educación, la Ciencia y la Cultura, 2017).

Desde un enfoque integral, la educación también debe asumirse a partir de sus características cualitativas, es decir, su calidad. Alcántara (2007) menciona que la calidad tiene diferentes acepciones y posibilidades. Concuerda con la disposición a una autoexigencia que, más que de fuera, procede del interior del sistema que busca su perfeccionamiento. En el caso de la educación, la calidad no radica únicamente en los distintos aspectos del proceso de enseñanza-aprendizaje, sino que concierne a la interacción entre todos los componentes del sistema. Una dimensión de la calidad que se asume desde la correlación entre objetivos, metas y resultados, se ocupa de la correspondencia entre lo que se ofrece $y$ lo que se entrega, o lo que se busca y lo que se logra. Para una institución académica, entonces, la calidad puede considerarse como el grado de aproximación a lo que ella ha definido como su misión propia.

\section{CONSIDERACIONES SOBRE LA CALIDAD EN LAS INSTITUCIONES DE EDUCACIÓN SUPERIOR}

A partir de una misión definida, uno de los principales retos que hoy guardan las Instituciones de Educación Superior (IES) en la búsqueda de alcanzar el cumplimiento de su razón de ser, radica en la dualidad con la que son percibidas por la sociedad: en primer lugar, se les aprecia por su cometido como generadoras de conocimiento y formadoras de profesionales capaces de transformar su entorno, a la vez que en su apresto para la resolución de diversas problemáticas sociales. Estas tareas, que de manera histórica han sido tamizadas bajo distintos criterios o parámetros, buscan garantizar el cumplimiento de cada misión institucional y su calidad; sin embargo, hoy día la sociedad reconoce a las IES también como organizaciones que gestionan capital humano y administran recursos materiales y financieros, teniendo el deber de hacerlo con transparencia, honestidad y eficiencia. Como ocurre con las acciones y el impacto que se produce en cualquier organismo que maneja recursos, las IES deben ser evaluadas a la luz de criterios $\mathrm{o}$ indicadores que reflejen su correcta administración. En la actualidad, hablar de calidad en la educación superior, y más si ésta es de carácter público, concierne no sólo al cumplimiento de sus funciones sustantivas, sino a una administración y gestión de los recursos eficiente y pertinente.

Para ponderarse la calidad en una institución educativa, se requiere de un sistema de evaluación con indicadores de desempeño que resulte relevante para los tomadores de decisiones, siendo esto fundamental en el caso de las entidades públicas que buscan optimizar los niveles de eficiencia y eficacia en el ejercicio de los recursos en todos los niveles, con la correspondiente rendición de cuentas (Bonnefoy y Armijo, 2005). 
Particularmente para las instituciones públicas de educación superior, la orientación de los indicadores se dirige a acrecentar la pertinencia de la educación a ese nivel, así como a aumentar la calidad de la misma en cada una de sus funciones y desde todos los actores que participan en ella (Sánchez et al., 2005).

La evaluación del desempeño en una institución educativa pública deberá determinarse a la luz de indicadores que reflejen tanto el grado de cumplimiento de sus tareas sustantivas como de la calidad con la que planean y administran sus recursos, bajo principios de eficiencia y transparencia.

\section{INDICADORES PARA LA EVALUACIÓN EN INSTITUCIONES PÚBLICAS}

La evaluación, en general, puede definirse como una acción sistemática respecto a la operación o impacto de un programa o política pública, en comparación con estándares, para contribuir a una determinada mejora o perfeccionamiento. El Instituto Latinoamericano de Planificación Económica y Social (ILPES) de la Comisión Económica para América Latina (CEPAL) clasifica la evaluación de la acción pública según su objeto o finalidad para la toma de decisiones, que se sintetiza en la evaluación de políticas públicas, de programas públicos, de gestión y resultado de las entidades públicas, y del desempeño individual. El Instituto plantea también que la evaluación puede ser externa, cuando la ejecuta alguna entidad independiente; interna, generalmente realizada por el órgano de control de la misma institución evaluada; ex ante, previamente a una determinada acción gubernamental; evaluación contingente, durante el proceso de ejecución; o ex post, una vez finalizada la acción por parte de la entidad pública. Independientemente del tipo de evaluación, el hecho de contar con indicadores favorece una apropiada planeación, facilita la detección de problemas de gestión, permite realizar ajustes en los procesos con el consecuente direccionamiento de políticas 0 reconsideración de trámites internos, coadyuva a expandir los niveles de transparencia en el ejercicio de los recursos públicos y constituye la base de los sistemas de reconocimiento al desempeño (Bonnefoy y Armijo, 2005). Por su parte la Organización para la Cooperación y el Desarrollo Económicos, OCDE (2010), menciona que la evaluación de resultados de gestión emplea metodologías basadas en el diseño de un sistema de indicadores que permite la rendición de cuentas respecto al ejercicio de los recursos y al grado de alcance de los objetivos establecidos, es decir, del nivel de resultados.

Si bien algunos de los primeros elementos teóricos sobre los indicadores de gestión provienen del sector privado, éstos se han ido adaptando a las instituciones públicas. En este sentido, Silva (2010) esboza el hecho de priorizar características de simplicidad, adecuación, oportunidad y validez en un lapso determinado. La primera se refiere a que el desarrollo y manejo de los indicadores sea poco costoso, optimizando recursos espaciales, temporales y financieros; la segunda, a la facilidad para amoldarse al hecho analizado y explicar los hallazgos; la oportunidad, alude a la capacidad de recolectar y analizar los datos en el tiempo requerido; $y$, finalmente, la validez, por el hecho de ser permanentes en un rango relevante de tiempo.

Si bien los sistemas de indicadores utilizados hasta el momento han demostrado su eficiencia al tratar de migrar prácticamente de manera literal a la educación superior, específicamente a la gestión de la 
administración en la educación pública, en ocasiones parecerían limitados ya que no atienden ciertas particularidades ni reflejan, del todo, las funciones que las IES realizan. No obstante, se reconoce que la existencia de indicadores representa una oportunidad de mejora y esto ha sentado las bases para evaluar de manera pertinente la gestión educativa.

Los indicadores, por lo tanto, se constituyen en un valor de referencia que permite la comparación entre las metas establecidas en la planeación y el resultado obtenido. Armijo (20I0), menciona que la CEPAL propone una clasificación de indicadores con base en las dimensiones del desempeño o según la etapa del proceso productivo o ámbito de medición. De acuerdo con las dimensiones del desempeño, un indicador de eficacia mide el grado de cumplimiento de un objetivo, sin considerar los recursos asignados, es decir, arroja una proporción. Los indicadores de eficiencia miden la relación entre los productos o servicios generados con respecto a los insumos o recursos utilizados, por ejemplo, los números o costos promedio por unidad de servicio. Un indicador de calidad mide atributos 0 capacidades de los bienes o servicios para satisfacer adecuadamente los objetivos de un programa público o los propósitos institucionales y suelen identificarse con los niveles de satisfacción de los usuarios, por lo que frecuentemente se relacionan con la percepción. Por otra parte, un indicador de economía mide la capacidad de un programa para generar $\circ$ ejercer recursos financieros en una institución. Estos indicadores, basados en las dimensiones del desempeño se complementan, según Pablos y Gil (2004), con los de equidad y los de gestión, analizando hasta qué punto existe disciplina en el ejercicio de un presupuesto universitario.
Lo anterior se ve complementado por Sánchez et al. (2005) al referir que, para instrumentar indicadores de evaluación de calidad en la educación superior, es necesario tomar decisiones para motivar un cambio y propiciar el convencimiento y compromiso de la alta dirección, el trabajo en equipo y la responsabilidad grupal para una mejora continua y la conformación del sistema de calidad, así como la actuación constante e involucramiento en la cultura de calidad en todos los niveles de las IES.

\section{LA CALIDAD Y LOS INDICADORES DE DESEMPEÑO EN LAS UNIVERSIDADES PÚBLICAS ESTATALES}

La calidad en la educación pública se ha considerado como el mejor referente para medir la eficiencia y eficacia del uso de los recursos destinados para tal fin, ya que representa la oportunidad de desarrollo y crecimiento de una sociedad, a la vez que se constituye como un detonador de cambio favorable para los individuos al generar oportunidades de acceso y formación que, de otra manera, difícilmente se podrían alcanzar.

Por la importancia de la educación pública, según Bonnefoy y Armijo (2005), la calidad de este servicio adquiere una dimensión relevante en función de la capacidad institucional para responder a los requerimientos en forma oportuna, precisa, continua y mediante canales accesibles. En este sentido, la UNESCO (1998), a través de la Conferencia Mundial sobre la Educación Superior, dio a conocer las misiones y funciones particulares de la educación, resumidas en 17 artículos cuyo propósito general ha sido contribuir al desarrollo sostenible y al mejoramiento de la sociedad, y que, de diversas maneras, aún prevalecen. En cuanto a 
la eficiencia de la gestión en las instituciones públicas de educación superior -a las que pertenecen las universidades públicas estatales (UPE)- el articulado muestra la exigencia de estrategias y capacidad de planeación a fin de garantizar la racionalidad con una perspectiva de futuro, siendo administradores receptivos y evaluadores de la propia eficacia de los procedimientos y resultados. Se propuso también, como objetivo primordial de la gestión, el óptimo cumplimiento de la misión institucional combinado con una visión social con competencias eficaces, en un marco de responsabilidad social fundada en la pertinencia, es decir, en qué tanto se adecuan los resultados entre lo que la sociedad espera y lo que está recibiendo. Estas consideraciones requieren de capacidad crítica e imparcialidad, un estricto apego a normas éticas, así como de acciones institucionales para erradicar la intolerancia, la violencia y el deterioro del medio ambiente, entre otros, contribuyendo al desarrollo de una sociedad integrada y motivada por el respeto hacia todos los seres humanos desde una plataforma de tolerancia y cooperación.

Las consideraciones sobre calidad en la educación pueden abordarse desde varias dimensiones como la filosófica, pedagógica, económica, cultural y social. Desde estos diversos ángulos, de acuerdo con Mungaray et al. (2006), estudios efectuados revelan que, en el caso de México, la asignación de ciertos recursos públicos, particularmente subsidios extraordinarios, ha sido efectiva al reconocer los avances de las UPE precisamente en materia de calidad, consolidación institucional y disminución de brechas de desempeño. En este sentido, la aplicación de indicadores da soporte a los procesos de planeación, particularmente al establecimiento de objetivos, metas y políticas, coadyuvando en la detección de problemas de una gestión como el uso ineficiente de recursos o el atraso en la entrega de productos o servicios requeridos; así mismo, permite comparativos entre los niveles de desempeño programados y los reales -con los respectivos ajustes-, favoreciendo el reconocimiento a las buenas prácticas a partir del compromiso con los resultados, y propiciando mayores niveles de transparencia y rendición de cuentas (Bonnefoy y Armijo, 2005).

Diferentes organismos internacionales como la UNESCO, la Organización para la Cooperación y el Desarrollo Económicos (OCDE), el Banco Mundial (BM) y el Banco Interamericano de Desarrollo (BID), además de diversas universidades a nivel mundial, dieron la pauta para la propuesta expuesta por Sánchez et al. (2005: I00-10I) que incorpora 48 indicadores para la evaluación de la calidad en la educación superior, incluyendo la de carácter público, agrupados en cinco criterios: factores institucionales y de organización; factores del contexto externo e interno; calidad de los insumos; calidad de los procesos; y calidad de los productos. Esto es una muestra de la necesidad de disponer de indicadores (índices) que sirvan para evaluar el quehacer de las UPE, a la vez que den rumbo y orientación a la implementación de políticas y acciones que fortalezcan el cumplimiento de sus tareas sustantivas. El diseño de sistemas de indicadores en la educación pública debe realizarse de acuerdo con las características propias, misión, visión y planes de desarrollo institucional, alineados con la política local y nacional en materia de educación superior; por ello, la construcción de dicho sistema debe tener en cuenta, además de los referentes o buenas prácticas, un análisis profundo a partir de cada plan estratégico institucional. 
La construcción de indicadores en el ámbito de la educación superior se conforma por diferentes etapas, no forzosamente consecutivas sino algunas simultáneas y que pueden retroalimentarse unas a otras. La cantidad de indicadores a construir, así como su monitoreo, dependerá del tipo y tamaño de la institución, de las metas concretas y cuantificables, de las prioridades de evaluación y los recursos disponibles, así como del propósito de la información a considerar (fines internos o externos). Aun cuando los resultados obtenidos pueden contrastarse con instituciones similares mediante un proceso de benchmarking, también pueden compararse al interior mismo respecto a resultados históricos, pero sobretodo en relación con lo planeado, teniendo como punto de comparación las propias metas organizacionales. En este sentido, la propuesta de la CEPAL referida por Bonnefoy y Armijo (2005), aplicable al sector público en general, se refiere a un ciclo basado en respuestas a diferentes cuestionamientos:

- ¿Cuáles objetivos estratégicos y metas serán evaluados?

- ¿Qué dimensiones del desempeño es conveniente medir (eficacia, eficiencia, calidad, resultados)?

- ¿Cuáles serán las áreas responsables por el desempeño logrado? ¿Hasta qué nivel de esa área llegará la evaluación?

- ¿Cuáles son los referentes para la comparación?

- ¿Cómo se relacionan las variables de medición?

- ¿Qué datos y antecedentes permitirán construir los indicadores? ¿Se cuenta con ellos de primera mano o se requiere una indagación más profunda?

- ¿Cómo se validarán los indicadores para constatar que efectivamente miden la gestión y los resultados?
- ¿Qué resultados se obtuvieron?

- ¿Quiénes deben conocer los resultados? ¿Cómo se les informará?

A la par de estas consideraciones, debe tenerse en cuenta, como lo propone Bass (2014), que un sistema de indicadores se conforma por un conjunto de datos medido y organizado de manera sistemática, que facilite la lectura y el consecuente análisis para los miembros de una organización. Podría afirmarse que un sistema de indicadores transita entre la exactitud científica y la información disponible bajo un modelo que permite el seguimiento de los objetivos propuestos, a la vez que identifica su grado de cumplimiento y/o la satisfacción para los usuarios, en este caso, relacionados con la institución de educación superior.

\section{EL DESEMPEÑO EN LA GESTIÓN}

\section{ADMINISTRATIVA-FINANCIERA EN LAS UNIVERSIDADES PÚBLICAS ESTATALES}

Actualmente no puede soslayarse la importancia de la medición de resultados, entendida como una relación de costo-beneficio que refleja los impactos arrojados por una inversión, en cualquiera de sus formas; así, la educación pública no es la excepción. Existen diferentes formas institucionales y sociales de rendición de cuentas que van desde la formalidad de las auditorías a los subsidios federales y estatales para la educación, hasta los diversos mecanismos de control interno que solicitan la rendición periódica de resultados a través, por ejemplo, de órganos colegiados. Es así como las funciones administrativas-financieras en las instituciones públicas de educación superior han adquirido una importancia fundamental como un detonador de las actividades sustantivas, pero también por la relevancia legal del correcto uso de los recursos. 
No augura éxito el hecho de contar con un proyecto educativo si el recurso utilizado no es transparentado $y$ apegado a la legalidad; de esta manera, la calidad de una acción no sólo recae en el ámbito académico, sino que también radica en la correcta aplicación de los recursos públicos.

Los Comités Interinstitucionales para la Evaluación de la Educación Superior, (CIEES) definen a la administración y la gestión en las instituciones de educación superior (IES) como:

...la manera mediante la cual los individuos se organizan y establecen una estructura para cumplir con la misión o fines establecidos en sus ordenamientos jurídicos, a través de la planeación de las acciones, su ejecución, control y evaluación, teniendo como marco la filosofía e identidad institucional (CIEES, 2018, p.4).

Evidentemente las UPES, como parte de las IES, comparten funciones administrativas y financieras que, en lo general, implican el aseguramiento de la calidad en la administración de sus recursos materiales y financieros; la coordinación de las actividades de obtención de recursos financieros; la promoción de técnicas adecuadas para la optimización y racionalización de los recursos institucionales; la creación y operación de mecanismos para incrementar los recursos financieros de la institución; la coordinación de los programas de capacitación, desarrollo y jubilación del personal académico y administrativo; la planeación, coordinación y evaluación de los procesos de compras, distribución de materiales y del control de los inventarios institucionales; la planeación, coordinación y evaluación de los procesos de ingresos y egresos financieros, así como el control presupuestal; la programación del mantenimiento de la infraestructura física; la administración de los convenios que suscribe de la institución; la formulación y diseño de las políticas en materia de recursos financieros; la optimización y racionalización de los recursos de la institución; la vinculación permanente con todas las entidades $y$ dependencias universitarias en función del logro de los objetivos institucionales; $y, \sin$ duda, la aplicación de mecanismos de transparencia y acceso a la información en materia administrativa y financiera (Universidad Autónoma del Estado de Hidalgo, s.f.).

En general, el objeto de evaluación de las funciones de una IES suele ser la gestión de la institución en su conjunto, más allá de los diversos campos del conocimiento que cubre. En particular, se evalúan las formas de gobierno y las normas que rigen la vida institucional, sus procesos de planeación y evaluación, sus estatutos orgánicos y administrativos, sus recursos financieros, los apoyos que orientan al ejercicio de sus funciones de docencia, investigación, extensión y difusión de la cultura, así como los mecanismos que le permiten vincularse con otras instituciones en el entorno nacional e internacional.

Los indicadores para la evaluación de las funciones administrativas $y$ financieras pueden ser tanto de desempeño como de percepción, siendo en general de carácter numérico cuyo resultado suele obtenerse como un porcentaje con base en diferentes variables. Cada indicador, individual y a la vez como parte de un sistema, tiene propiedades que lo legitiman; así lo presentan Carot et al. (20I2) cuya propuesta radica en el cumplimiento de los criterios de pertinencia, validez, fiabilidad, comparabilidad, comunicabilidad, resistencia a la manipulación, factibilidad, economía y vinculación con los objetos. Se busca además que, como parte de un sistema, se guarden las cualidades de precisión, utilidad, 
completitud y simplicidad. Esto significa que dicho sistema refleja los rasgos distintivos de lo que se pretende describir, así como su conveniencia; que no hay lugar a ambigüedad, sino que el usuario puede medir o interpretar sin conflicto; que refleja la totalidad del objeto de medición, donde cada elemento representa algún aspecto del sistema; que además de ser completo y pertinente, es a la vez sencillo; y finalmente, que el sistema es de utilidad pues constituye una herramienta de valor para la toma de decisiones del ámbito administrativo-financiero en favor de la institución.

Para la construcción de índices para la evaluación del desempeño administrativo y financiero, puede tenerse como referente la metodología que proponen Saisana y Tarantola (2002), cuyas etapas consisten en decidir el atributo que se quiere medir, seleccionar los indicadores simples, ponderar la calidad de los datos, evaluar la relación existente entre indicadores simples, normalizar datos para permitir comparabilidad, seleccionar los métodos de agregación y ponderación de los indicadores simples y, finalmente, evaluar la calidad de cada indicador compuesto o índice.

Una vez construidos los indicadores compuestos, se procede al desarrollo de un modelo, lo que brinda la posibilidad de contar con datos sobre los niveles de cumplimiento de los propósitos planteados y los resultados esperados por cada programa o línea de acción relativos a las funciones tanto administrativas como financieras, así como de enriquecer los análisis en cuanto al uso de los recursos y transparencia en su ejercicio.

\section{CONCLUSIÓN}

Como ocurre en cualquier organismo que maneja recursos públicos, actualmente las UPE deben ser evaluadas mediante criterios que reflejen su correcta administración. Se considera que, para el desarrollo de indicadores, deberán clarificarse las dimensiones de la evaluación y su pertinencia, así como el uso de la información, para efectivamente transitar a un esquema que pondere eficientemente el desempeño en la gestión de las universidades públicas estatales.

El diseño de un sistema de indicadores se determina de acuerdo con las características, filosofía y planes de desarrollo institucional, alineados con la política local y nacional en materia de educación superior, con la finalidad de medir y evaluar el desempeño, teniendo como marco el comportamiento ético, la capacidad crítica y la imparcialidad.

Hablar de calidad en la educación superior, particularmente si es de carácter público, atañe no únicamente al cumplimiento de las funciones sustantivas, sino a una gestión eficiente y pertinente de los recursos institucionales. Lo anterior deriva en la exigencia de contar con indicadores o índices para la evaluación de la gestión administrativa-financiera, agregados en un sistema completo, pertinente, sencillo y de valor, que permita ponderar el quehacer de cada UPE en aras de un desarrollo institucional integral.

\section{REFERENCIAS}

Alcántara, A. (2007). Dimensiones de la calidad en educación superior. Reencuentro, (50). http://www.redalyc.org/pdf/340/34005004.pdf

Armijo, M. (20I0). Lineamientos metodológicos para la construcción de indicadores de desempeño. Montevideo: ONU. https://www.cepal.org/ilpes/noticias/paginas/0/35 060/indicadoresdesempenoarmijom.pdf 
Bas, M. (2014). Estrategias metodológicas para la construcción de indicadores compuestos en la gestión universitaria. Valencia: Universitat Politécnica de Valencia.

Bonnefoy, J. C. \& Armijo, M. (2005). Indicadores de desempeño en el sector público. Santiago: ONU. https://repositorio.cepal.org/bitstream/handle/ I I 362/56 I I/S05900 es.pdf

Carot, J. M., Henríquez, P., Haug, G., Mora, J. G., Ristoff, D., Vidal, J., Vila, L., y González, E. (20I2). Sistema Básico de Indicadores para la Educación Superior de América Latina. Editorial Universitat Politécnica de Valencia, Valencia.

https://atenea.epn.edu.ec/bitstream/25000/365/I/ Sistema-Basico-de-Indicadores-para-laEducacion-Superior-de-America-Latina-Marzo20I2-Version-completa.pdf

Centro Interuniversitario de Desarrollo (20I2). Aseguramiento de la calidad en Iberoamérica. Santiago: CINDA. https://cinda.cl/wpcontent/uploads/2012/05/aseguramiento-de-lacalidad-en-iberoamerica-educacion-superiorinforme-2012.pdf

Comités Interinstitucionales para la Evaluación de la Educación Superior (2018). Principios y estándares para la acreditación de instituciones de educación superior de México.

http://www.ciees.edu.mx/documentos/principiosy-estandares-para-la-acreditacion-deinstituciones-de-educacion-superior-20 I8.pdf

Instituto Internacional de Unesco para la Educación Superior en América Latina y el Caribe (2017). Educación Superior y Sociedad. 22. https://www.iesalc.unesco.org/ess/index.php/ess3 larticle/view/36/36

Mungaray, A., Ocegueda, M. T.; Moctezuma, P. \& Ocegueda, J. M. (20I6). La calidad de las Universidades Públicas Estatales de México después de 13 años de subsidios extraordinarios. Revista de la Educación Superior. 45(I), 67-93.

Organización de las Naciones Unidas para la Educación, la Ciencia y la Cultura (1998). Declaración mundial sobre la educación superior en el siglo XXI: Visión y acción. Conferencia mundial sobre la educación superior. París: UNESCO. http://www.unesco.org/education/educprog/wch e/declaration_spa.htm\#declaracion
Organización de las Naciones Unidas para la Educación, la Ciencia y la Cultura. (20I7). El derecho a la educación. Social and Human Sciences. http://www.unesco.org/new/es/social-andhumansciences/themes/advancement/networks/larno/rig ht-to-education/

Organización para la Cooperación y el Desarrollo Económicos. (2010). Estándares de calidad para la evaluación del desarrollo. París: OCDE. https://www.oecd.org/dac/evaluation/dcdndep/46 297655.pdf

Pablos, L. \& Gil, M. (2004). Algunos indicadores de equidad, eficiencia y eficacia del sistema de financiación de la Universidad. Documentos de Trabajo de la Facultad de Ciencias Económicas y Empresariales. (19). https://eprints.ucm.es/6842///0419.pdf

Peña Frade, N. (20I5). Sistema de aseguramiento de la calidad de la educación superior. Una política pública que merece otra reflexión. Criterio Jurídico Garantista. 7(I2). I I4-I45. Bogotá: Universidad Autónoma de Colombia. http://revistas.fuac.edu.co/index.php/criteriojuridi cogarantista/article/download/468/448

Saisana, M. y Tarantola, S. (2002). State-of-the-art report on current methodologies and practices for composite indicator development (Vol. 2I4). Ispra: European Commission, Joint Research Centre, Institute for the Protection and the Security of the Citizen, Technological and Economic Risk Management Unit.

Sánchez, J.; Vázquez, M., Gándara, R. \& González, G. (2005). Criterios e indicadores para la evaluación de la calidad en las instituciones de educación superior. Mercados y Negocios. 12(6). http://revistascientificas.udg.mx/index.php/MYN/a rticle/viewFile/5013/4682

Silva, D. (20I0). Teoría de indicadores de gestión y su aplicación práctica. Universidad Militar Nueva Granada. http://www.umng.edu.co/documents/I0162//299 3I7/ART_29.pdf

Universidad Autónoma del Estado de Hidalgo (s.f.). Coordinación de Administración y Finanzas Funciones. https://www.uaeh.edu.mx/adminyserv/gesuniv/div _admonfin/funciones.html 